\title{
Femtosecond Laser Micromachining of Fabry-Pérot Interferometers for Magnetic Field Sensing
}

\author{
João M. Maia ${ }^{1,2 *}$, Vitor A. Amorim ${ }^{1,2}$, Duarte Viveiros ${ }^{1,2}$, and P. V. S. Marques ${ }^{1,2}$ \\ ${ }^{1}$ CAP - Centre for Applied Photonics, INESC TEC, Rua do Campo Alegre 4150-179, Porto, Portugal \\ ${ }^{2}$ Department of Physics and Astronomy, Faculty of Sciences of University of Porto, Rua do Campo Alegre 4169-007, Porto, Portugal
}

\begin{abstract}
Fs-laser micromachining is a high precision fabrication technique that can be used to write novel three-dimensional structures, depending on the nature of light-matter interaction. In fused silica, the material modification can lead to (i) an increase of the refractive index around the focal volume, resulting in the formation of optical circuits, or (ii) an enhancement of the etch rate of the laser-affected zones relative to the pristine material, leading to a selective and anisotropic etching reaction that enables fabrication of microfluidic systems. Here, both effects are combined to fabricate a Fabry-Pérot interferometer, where optical waveguides and microfluidic channels are integrated monolithically in a fused silica chip. By filling the channel with a magnetic fluid whose refractive index changes with an external magnetic field, the device can be used as a magnetic field sensor. A linear sensitivity of $-0.12 \mathrm{~nm} / \mathrm{mT}$ is obtained in the $5.0 \pm 0.5$ to $33.0 \pm 0.5 \mathrm{mT}$ range, with the field being applied parallel to the light propagation direction.
\end{abstract}

\section{Introduction}

Ferrofluids are stable colloidal suspensions of singledomain magnetic nanoparticles dispersed in an aqueous or nonpolar solvent. Under an external magnetic field, the nanoparticles start to agglomerate in a chain-like structure oriented with the field direction, thereby changing the fluid's optical properties: refractive index, birefringence, dichroism, field-dependent transmission [1, 2]. Taking advantage of this phenomenon, several fiber-based magnetic field sensors for measurement of field magnitude or orientation have been demonstrated [3-5].

So far, photonic devices based on ferrofluids have only been used in planar systems for light modulation applications [6]. Compared to the existent fiber-based sensors, planar devices offer the chance of simultaneous detection of the field strength and its direction, made available not only due the ferrofluid properties but also by the advances made in fs-laser micromachining [7]. Ergo, this paper discusses the fabrication of Fabry-Pérot interferometers in fused silica by fs-laser micromachining and their response to magnetic field variations.

\section{Experimental}

The sensor's operation is based on wave interference, and since the contrast between the ferrofluid and fused silica is small, two-wave interference is enough to describe the process. The sensor is also designed as a reflective structure to maximize visibility. The reflected intensity $\left(I_{r}\right)$ is given by:

$$
I_{r}=I_{1}+I_{2}+2 \sqrt{I_{1} I_{2}} \cos \delta
$$

where $I_{1}$ and $I_{2}$ are the intensities of the light beams reflected at the first and second interfaces, respectively, and have an impact on visibility. The condition for constructive interference is determined by the phase difference $(\delta)$ between both waves which relies on the cavity length and the ferrofluid refractive index. The latter further depends on the direction and magnitude of the magnetic field. As a result, the proposed device can be used as a magnetic field sensor.

\subsection{Fabrication}

A fiber amplified fs-laser system, emitting a second harmonic beam at $515 \mathrm{~nm}$ with an approximate pulse duration of $250 \mathrm{fs}$ at $500 \mathrm{kHz}$, was used to write the interferometer. The laser beam was focused inside a fused silica substrate (Suprasil 1) with a 0.55 numerical aperture aspherical lens mounted in a vertical piezostage. The substrate was mounted on X-Y air-bearing linear stages and translated in relation to the beam focus. The optical waveguide and microfluidic channel were written sequentially to guarantee perfect alignment between both.

A $1.2-\mathrm{cm}$ long waveguide was written $55 \mu \mathrm{m}$ below the silica surface at a speed of $50 \mu \mathrm{m} / \mathrm{s}$, pulse energy of $75 \mathrm{~nJ}$ and with the polarisation of the laser beam parallel to the writing direction. These parameters yield low scattering, which affects the Fabry-Pérot's signal-to-noise ratio. The microfluidic channel was written from bottomto-top by stacking multiple laser tracks with a horizontal and vertical spacing of 2 and $3 \mu \mathrm{m}$ respectively, at a speed of $500 \mu \mathrm{m} / \mathrm{s}$, pulse energy of $80 \mathrm{~nJ}$ and with the beam's polarisation perpendicular to the scanning direction. In these conditions, the channel has low surface roughness.

\footnotetext{
* Corresponding author: joao.m.maia@inesctec.pt
} 
Etching was done in an ultrasonic bath by immersing the substrate in a $10 \%$ hydrofluoric acid solution for 240 minutes. The channel has a final height of $90 \mu \mathrm{m}$ and a tapered cross-section; the cavity length is $35 \mu \mathrm{m}$. The waveguide and the channel wall are distanced by $8 \mu \mathrm{m}$.

The substrate's facet was polished to improve light coupling. To inject the ferrofluid and to seal the device, a PDMS layer with two inlets/outlets was permanently bonded to the silica substrate. Sealing the device is necessary as the ferrofluid rapidly evaporates. A waterbased ferrofluid (EMG507, Ferrotec Inc.), composed by $\mathrm{Fe}_{3} \mathrm{O}_{4}$ magnetic nanoparticles with average diameter of $10 \mathrm{~nm}$ in a volume concentration of $0.4-1.1 \%$ was used.

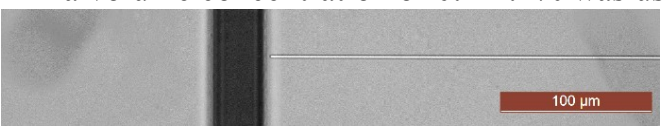

Fig. 1. Top-view of the optical waveguide orthogonally aligned with the microfluidic channel.

\section{Results and Discussion}

To measure the magnetic field, light from a broadband source is injected into an optical circulator and coupled into a single-mode fiber (SMF-28) that is butt-coupled to the waveguide. Light reflected in the Fabry-Pérot is transmitted back into the circulator, before being detected by an optical spectrum analyser. The substrate was mounted on a 5-axis stage for a precise alignment of the optical fiber with the waveguide. Index matching was used to minimize Fresnel reflections at the facet. A permanent magnet placed close to the substrate generates a magnetic field parallel to the light propagation direction. Its magnitude can be controlled by changing the distance between the magnet and the substrate. The measurements were made right after the ferrofluid was injected to prevent the nanoparticles from settling in the channel bottom. The temperature remained at $21.0 \pm 0.5^{\circ} \mathrm{C}$ and, in turn, thermally-induced index variations can be neglected.

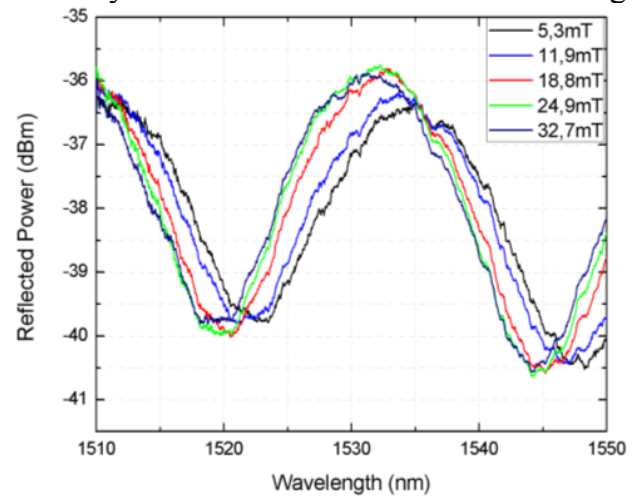

Fig. 2. Reflected spectrum at different field magnitudes.

As can be observed in Fig. 2, the interference spectrum shows a blue-shift when the field strength increases, indicating that the fluid's refractive index is decreasing. This is contradictory to what is expected [1-4] and can be explained by the fact that the nanoparticles are attracted towards the magnet and, consequently, they exit the cavity leading to a smaller concentration of nanoparticles and a decreasing refractive index. The low visibility of around
$4 \mathrm{~dB}$ is due to the high optical absorption coefficient of the ferrofluid $(\approx 20 \mathrm{~dB}$ per $100 \mu \mathrm{m})$. After smoothing the measured spectra, we retrieved the peak wavelength for each field magnitude (Fig. 3). A linear sensitivity of $-0.12 \mathrm{~nm} / \mathrm{mT}$ was obtained across the entire measured range. The use of ferrofluids with higher concentration of magnetic nanoparticles can enhance the sensitivity [3, 4].

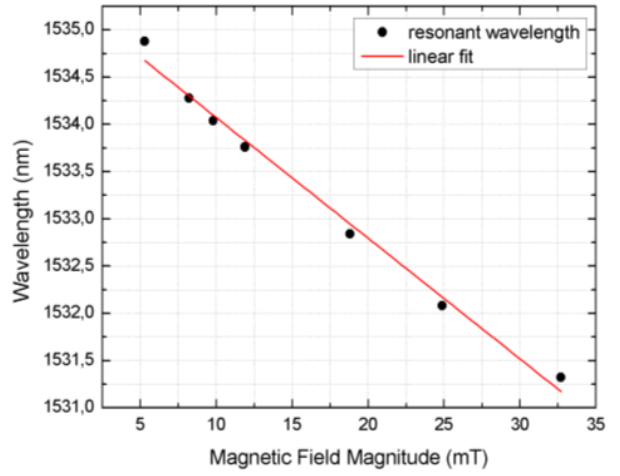

Fig. 3. Peak wavelength as a function of field magnitude.

\section{Conclusion}

Due to the tunable refractive index property of ferrofluids, an optofluidic sensor for magnetic field detection was fabricated by fs-laser micromachining. Preliminary results show that the sensor responds to field variations with a sensitivity of $-0.12 \mathrm{~nm} / \mathrm{mT}$ from $5.0 \pm 0.5$ to $33.0 \pm 0.5 \mathrm{mT}$. Due to the $3 \mathrm{D}$ capabilities of fs-laser micromachining, other functionalities, enabled by the ferrofluid properties, can be envisioned: measurement (i) of field orientation by writing two other waveguides along the remaining spatial directions, or (ii) of time-varying fields through spectral filtering with Bragg gratings.

This work was supported by Fundação para a Ciência e Tecnologia through grant no. SFRH/BD/133095/2017 and by project "NanoSTIMA: Macro-to-Nano Human Sensing: Towards Integrated Multimodal Health Monitoring and Analytics/NORTE-01-0145-FEDER-000016", financed by the North Portugal Regional Operational Programme (NORTE 2020), under the PORTUGAL 2020 Partnership Agreement, and through the European Regional Development Fund (ERDF).

\section{References}

1. S.Y. Yang, J.J. Chieh, H.E. Horng, C.-Y. Hong, H.C. Hang, Appl. Phys. Lett. 84, 25 (2004)

2. Z. Di, X. Chen, S. Pu, X. Hu, Y. Xia, Appl. Phys. Lett. 89, 21 (2006)

3. R. Blue, A. Duduś, D. Uttamchandani, IEEE J. Sel. Topics Quantum Elceton. 22, 2 (2016)

4. D. Homa, G. Pickrell, Sensors 14, 3 (2014)

5. Z. Jiang, J. Dong, S. Hu, Y. Zhang, Y. Chen, Y. Luo, W. Zhu, W. Qiu, H. Lu, H. Guan, Y. Zhong, J. Yu, J. Zhang, Z. Chen, Opt. Lett. 43, 19 (2018)

6. Y. Gu, F. Bragheri, G. Valentino, K. Morris, N. Bellini, R. Osellame, Appl. Opt. 54, 6 (2015)

7. K. Sugioka, J. Xu, D. Wu, Y. Hanada, Z. Wang, Y. Cheng, K. Midorikawa, Lab Chip 14, 18 (2014) 